Commun. math. Phys. 7, 99-111 (1968)

\title{
Algebras of Observables with Continuous Representations of Symmetry Groups*
}

\author{
KarL KraUs \\ Institut für Theoretische Physik (I) der Philipps-Universität \\ Marburg/Lahn, Germany
}

Received December 18, 1966/August 30, 1967

\begin{abstract}
Concrete $C^{*}$-algebras, interpreted physically as algebras of observables, are defined for quantum mechanics and local quantum field theory.

A quantum mechanical system is characterized formally by a continuous unitary representation up to a factor $U_{g}$ of a symmetry group $\mathfrak{G}$ in Hilbert space $\mathfrak{S}$ and a von Neumann algebra $\mathfrak{R}$ on $\mathfrak{S}$ invariant with respect to $U_{g}$. The set $\mathfrak{A}$ of all operators $X \in \Re$ such that $U_{g} X U_{\sigma}^{-1}$, as a function of $g \in \mathfrak{F}$, is continuous with respect to the uniform operator topology, is a $C^{*}$-algebra called the algebra of observables. The algebra $\mathfrak{R}$ is shown to be the weak (or strong) closure of $\mathfrak{A}$.

In field theory, a unitary representation up to a factor $U(a, \Lambda)$ of the proper inhomogeneous Lorentz group $\mathfrak{S}$ and local von Neumann algebras $\mathfrak{R}_{C}$ for finite open space-time regions $C$ are assumed, with the usual transformation properties of $\Re_{c}$ under $U(a, \Lambda)$. The collection of all $X \in \mathfrak{R}_{c}$ giving uniformly continuous functions $U(a, \Lambda) X U^{-1}(a, \Lambda)$ on $\mathfrak{G}$ is then a local $C^{*}$-algebra $\mathfrak{A}_{c}$, called the algebra of local observables. The algebra $\mathfrak{A}_{C}$ is again weakly (or strongly) dense in $\mathfrak{R}_{C}$. The normclosed union $\mathfrak{A}$ of the $\mathfrak{A}_{C}$ for all $C$ is called algebra of quasilocal observables (or quasilocal algebra).

In either case, the group $\mathfrak{G}$ is represented by automorphisms $\mathbf{V}_{g}$ resp. $\mathbf{V}(a, \Lambda)$ - with $\mathbf{V}_{g} X=U_{g} X U_{\sigma}^{-1}$ - of the $C^{*}$-algebra $\mathfrak{A}$, and this is a strongly continuous representation of $\mathfrak{G}$ on the Banach space $\mathfrak{A}$. Conditions for $\mathbf{V}(a, \Lambda)$ can then be formulated which correspond to the usual spectrum condition for $U(a, \Lambda)$ in field theory.
\end{abstract}

\section{Introduction and Summary}

In quantum mechanics, physical quantities (observables) are represented by Hermitean operators $A$ on a certain Hilbert space $\mathfrak{S}$. If moreover these observables are suitably selected, they can be represented by bounded operators $A$. Implicitely or explicitely, most theoretical investigations also assume the inverse: Any bounded Hermitean operator $A$ on $\mathfrak{G}$ compatible with the superselection rules (i.e., commuting with all "superobservables") of the theory is supposed to represent a physical observable. The set of observables then coincides with the set of all Hermitean operators of a certain von Neumann algebra $\mathfrak{R}$. In field theory, the introduction of local von Neumann algebras $\mathfrak{R}_{C}$ for all

\footnotetext{
* Work supported in part by the Deutsche Forschungsgemeinschaft.
} 
finite open space-time regions $C$ leads to a similar assumption: The Hermitean operators $A$ of $\Re_{C}$ are supposed to represent possible measurements in $C$.

But the sets of observables so defined are likely much too large. A physical system without superselection rules, for instance, is intuitively expected to have much fewer different physical observables (i.e., measuring apparatus which can actually be constructed, at least in principle) than Hermitean operators on the corresponding Hilbert space. Under these circumstances there should exist, besides Hermiticity, some extra properties which would characterize the "true" observables in the von Neumann algebras $\mathfrak{R}$ resp. $\mathfrak{R}_{C}$.

As a purely formal attempt to formulate such an additional property of observables, we are going to postulate a sufficiently regular transformation behavior with respect to symmetry transformations of the physical system: If $A$ is any observable and $U_{g}$ the given strongly continuous unitary representation up to a factor of the symmetry group $\mathfrak{G}$ in Hilbert space $\mathfrak{S}$, then $U_{g} A U_{g}^{-1}$, as a function of the group element $g \in(\mathfrak{S}$, has to be continuous with respect to the uniform operator topology (norm topology). Vice versa, any Hermitean operator $A$ in $\mathfrak{R}$ resp. $\mathfrak{R}_{C}$ with this property is assumed to be an observable. In field theory, the group $\mathfrak{G}$ is identified with the inhomogeneous Lorentz group.

The set of operators $A+i B$ with observables $A$ and $B$ from $\mathfrak{R}$ is then a concrete $C^{*}$-algebra $\mathfrak{A}$, called algebra of observables. Similarly, the $A+i B$ with observables $A, B$ from a local $\mathfrak{R}_{C}$ form a $C^{*}$-subalgebra $\mathfrak{A}_{C}$ of $\mathfrak{R}_{C}$, called the algebra of local observables belonging to the spacetime region $C$. In either case, the algebra of observables $\mathfrak{A}$ resp. $\mathfrak{A}_{C}$ is weakly (or strongly, ultraweakly, ultrastrongly) dense in the corresponding von Neumann algebra $\mathfrak{R}$ resp. $\mathfrak{R}_{C}$. The union of the local $\mathfrak{A}_{C}$ for all finite open regions $C$ yields, after uniform closure, a concrete $C^{*}$-algebra $\mathfrak{A}$, the so called algebra of quasilocal observables (or shortly, quasilocal algebra). This quasilocal algebra is the field theoretic analogue to the algebra of observables $\mathfrak{A}$ for a quantum mechanical system.

A similar mathematical structure for the observables of field theory was proposed by HAAG and KASTLER [1]. In ref. [1], however, the concrete $C^{*}$-algebra $\mathfrak{A}$ defined above and its local subalgebras $\mathfrak{A}_{C}$ (i.e., $C^{*}$-algebras realized as operator algebras on Hilbert space) are replaced by abstract $C^{*}$-algebras.

The quantum mechanical algebra of observables $\mathfrak{A}$ contains in particular all completely continuous operators in 2 . On the contrary, the quasilocal algebra $\mathfrak{A}$ cannot contain any completely continuous operator (except zero), because the local von Neumann algebras $\mathfrak{R}_{C}$ do not contain such operators. 
The transformations $\mathbf{V}_{g}$ defined by

$$
\mathbf{V}_{g} X=U_{g} X U_{g}^{-1} \text { for all } X \in \mathfrak{A}
$$

are automorphisms of $\mathfrak{A}$ and provide a representation of the symmetry group $\mathfrak{G}$. By construction, the bounded linear operator $\mathbf{V}_{g}$ on the Banach space $\mathfrak{A}$ is a strongly continuous function of the group element $g$. Conditions corresponding to the usual spectrum postulate of field theory can then be formulated purely in terms of the quasilocal algebra $\mathfrak{A}$ and the representation $\mathbf{V}_{g}=\mathbf{V}(a, \Lambda)$ of the inhomogeneous Lorentz group $\mathfrak{S}$ on $\mathfrak{A}$ (Montvay [2], Doplicher [3]).

\section{The Quantum Mechanical Algebra of Observables}

Let $\mathfrak{R}$ be a von Neumann algebra on a Hilbert space $\mathfrak{S}$ with the following property: A suitable decomposition of $\mathfrak{g}$ into the discrete direct sum $\mathfrak{G}=\sum_{s} \oplus \mathfrak{G}_{s}$ of "coherent subspaces" $\mathfrak{S}_{s}$ reduces $\mathfrak{R}$ to the direct sum $\mathfrak{R}=\sum_{s} \oplus \mathfrak{Z}\left(\mathfrak{G}_{s}\right)$ of the algebras $\mathfrak{Z}\left(\mathfrak{G}_{s}\right)$ of all bounded operators on $\mathfrak{G}_{s}$. This corresponds physically to the existence of an Abelian set of superobservables, all with discrete spectrum, which are simultaneously diagonalized by the decomposition of $\mathfrak{g}, \mathfrak{R}$ being the set of all operators commuting with these superobservables. In the simplest case, without superselection rules, $\mathfrak{\imath}$ is simply the algebra $\mathfrak{Z}(\mathfrak{G})$ of all bounded operators on $\mathfrak{S}$.

Furthermore, let $U_{g}$ be a unitary, strongly continuous representation up to a factor of a connected Lie group $\mathfrak{G}$, the symmetry group of the physical system described in $\mathfrak{G}$ :

$$
\begin{gathered}
U_{g} U_{h}=\omega(g, h) U_{g h} \quad \text { with unitary operators } \quad \omega(g, h) \in \mathfrak{R}^{\prime}, \\
U_{e}=1 \quad(e=\text { unit element of } \mathfrak{F}), \\
\lim _{g \rightarrow g_{0}} U_{g}=U_{g_{0}} \quad \text { (strong operator topology). }
\end{gathered}
$$

For $\mathfrak{R}=\mathfrak{3}(\mathfrak{S})$ the unitary operators $\omega(g, h)$ are simply complex numbers of unit modulus. In the more general case considered here, $\omega(g, h) \in \mathfrak{R}^{\prime}$ is the appropriate generalization for representations "up to a factor". Moreover the $U_{g}$ must leave invariant all coherent subspaces $\mathfrak{S}_{s}$, from which follows $U_{g} \in \Re$ and

$$
U_{g} \mathfrak{R} U_{g}^{-1}=\mathfrak{R} \text { for all } g \in \mathfrak{G} .
$$

The representation up to a factor $U_{g}$ induces a representation $\mathbf{V}_{g}$ of $\mathfrak{G}$ by symmetric automorphisms of $\mathfrak{R}$ with

$$
\mathbf{V}_{g} X=U_{g} X U_{g}^{-1} \text { for all } X \in \Re,
$$


i.e.,

$$
\begin{gathered}
\mathbf{V}_{g} \mathbf{V}_{h}=\mathbf{V}_{g h}, \quad \mathbf{V}_{e}=\mathbf{1} \quad \text { (the identity automorphism) } \\
\mathbf{V}_{g}(\lambda X+\mu Y)=\lambda \mathbf{V}_{g} X+\mu \mathbf{V}_{g} Y, \\
\mathbf{V}_{g} X Y=\left(\mathbf{V}_{g} X\right)\left(\mathbf{V}_{g} Y\right), \quad \mathbf{V}_{g} X^{*}=\left(\mathbf{V}_{g} X\right)^{*}, \quad \mathbf{V}_{g} \mathbf{l}=\mathbf{1}, \\
\left\|\mathbf{V}_{g} X\right\|=\|X\|,
\end{gathered}
$$

and the strong continuity of $U_{g}$ immediately implies

$$
\lim _{g \rightarrow g_{0}} \mathbf{V}_{g} X=\mathbf{V}_{g_{0}} X \quad \text { (strong operator topology). }
$$

A subset $\mathfrak{A}$ of operators $X \in \Re$ will be defined by the continuity requirement

i.e.,

$$
\lim _{g \rightarrow g_{0}} \mathbf{V}_{g} X=\mathbf{V}_{g_{0}} X \quad \text { (uniform operator topology) }
$$

$$
\lim _{g \rightarrow g_{0}}\left\|\mathbf{V}_{g} X-\mathbf{V}_{g_{0}} X\right\|=0
$$

which is stronger than (1). As evident from the representation property of $\mathbf{V}_{g}, \mathfrak{A}$ contains with $X$ also all $\mathbf{V}_{g} X$, and (2) is equivalent to

$$
\lim _{g \rightarrow e}\left\|\mathbf{V}_{g} X-X\right\|=0 \text {. }
$$

Because of $\mathbf{V}_{g} \mathbf{l}=\mathbf{1}$ or, more generally, $\mathbf{V}_{g} S=S$ for all operators $S \in \mathfrak{R}^{\prime}$, the unit operator as well as all "bounded functions of superobservables", i.e., all $S \in \mathfrak{R}^{\prime}$, belong to $\mathfrak{A}$.

With $X$ and $Y$ also the operators $\lambda X+\mu Y, X Y$, and $X *$ belong to $\mathfrak{A}$ : They lie in $\mathfrak{R}$ because $\mathfrak{R}$ is a symmetric algebra, and the condition (3) can be verified using simple estimates of the operator norm. Moreover, $\mathfrak{A}$ contains all its accumulation points with respect to the uniform operator topology: They lie in $\mathfrak{R}$ because $\mathfrak{R}$ is uniformly closed, and ( 3 ) is again easy to show. Thus the following theorem is proved:

Theorem 1. $\mathfrak{A}$ is a symmetric, uniformly closed algebra of operators on Hilbert space $\mathfrak{G}$, i.e., (using the terminology of ref. [1]) a concrete $C^{*}$-algebra.

In what follows $\mathfrak{A}$ is called the algebra of observables. Hermitean operators $A \in \mathfrak{A}$ will be interpreted physically as observables of the given system. They are uniquely characterized in $\mathfrak{R}$ by Hermiticity and condition (3). Any operator $X \in \mathfrak{A}$ can be represented as $A+i B$ with observables $A=\frac{1}{2}\left(X^{*}+X\right)$ and $B=\frac{i}{2}\left(X^{*}-X\right)$.

Theorem 2. Any completely continuous operator $X \in \mathfrak{R}$ belongs to $\mathfrak{A}$, i.e., any completely continuous Hermitean operator in $\mathfrak{R}$ is an observable.

Proof. It is sufficient to show $A \in \mathfrak{A}$ for any completely continuous Hermitean operator $A \in \Re$ because then, from any completely continuous $X \in \mathfrak{R}$, one gets Hermitean

$$
A=\frac{1}{2}\left(X^{*}+X\right) \in \mathfrak{A}, \quad B=\frac{i}{2}\left(X^{*}-X\right) \in \mathfrak{A},
$$


and therefore one has $X=A+i B \in \mathfrak{A}$. A completely continuous Hermitean $A \in \Re$ has a spectral representation $A=\sum_{i} \lambda_{i} P_{\varphi_{i}}$ with $P_{\varphi_{i}} \in \mathfrak{P}$ and $\lim _{i \rightarrow \infty} \lambda_{i}=0$, and this implies $\lim _{n \rightarrow \infty}\left\|A_{n}-A\right\|=0$ with $A_{n}=\sum_{i=1}^{n} \lambda_{i} P_{\varphi_{i}} \in \mathfrak{R}$. Because $\mathfrak{A}$ is a uniformly closed linear space, it suffices to prove $P_{\varphi_{i}} \in \mathfrak{R}$. All one-dimensional projections $P_{\varphi} \in \mathfrak{R}$, however, belong to $\mathfrak{A}$. Indeed, the relation (3) for any one-dimensional $P_{\varphi}$, i.e.,

$$
\lim _{g \rightarrow e}\left\|\mathbf{V}_{g} P_{\varphi}-P_{\varphi}\right\|=\lim _{g \rightarrow e}\left\|P_{U_{g} \varphi}-P_{\varphi}\right\|=0,
$$

follows from the strong continuity of $U_{g}$, i.e., from

$$
\lim _{g \rightarrow e}\left\|U_{g} \varphi-\varphi\right\|=0,
$$

if one uses the simple estimate

with $\psi=U_{g} \varphi$.

$$
\left\|P_{\psi}-P_{\varphi}\right\| \leqq 2\|\psi-\varphi\|
$$

Theorem 3. The algebra of observables $\mathfrak{A}$ is dense in the von Neumann algebra $\Re$ with respect to the weak (or strong, ultraweak, ultrastrong) operator topology, or equivalently: $\mathfrak{H}^{\prime \prime}=\mathfrak{R}$.

Proof. According to Dixmier [4] (Chap. I, §3, No. 4, Corollaire 1), the weak, ultraweak, strong, and ultrastrong closure of $\mathfrak{A}$ all coincide with the von Neumann algebra $\mathfrak{A}^{\prime \prime}$ generated by $\mathfrak{A}$. Therefore it is enough to show $\mathfrak{U}^{\prime \prime}=\mathfrak{R}$. From $\mathfrak{A} \subset \mathfrak{R}$ the relation $\mathfrak{U}^{\prime \prime} \subset \mathfrak{R}$ is obvious, and thus only $\mathfrak{R} \subset \mathfrak{Q}^{\prime \prime}$ remains to be proved.

The projectors $P_{\mathfrak{S}_{s}} \in \mathfrak{R}^{\prime}$ onto the coherent subspaces $\mathfrak{S}_{s}$ belong to $\mathfrak{A}$ and thus to $\mathfrak{A}^{\prime \prime}$, since $\mathfrak{P}^{\prime} \subset \mathfrak{A}$. Furthermore, because of $\mathfrak{A} \subset \mathfrak{R}$, they belong to $\mathfrak{I}^{\prime} \supset \mathfrak{R}^{\prime}$ too. Thus they lie in the center of the von Neumann algebra $\mathfrak{A}^{\prime \prime}$ as well as in that of $\mathfrak{R}$, and one has, besides $\mathfrak{R}=\sum_{s} \oplus \mathfrak{B}\left(\mathfrak{G}_{s}\right)$, also the direct sum decomposition $\mathfrak{I}^{\prime \prime}=\sum_{s} \oplus\left(\mathfrak{Q}^{\prime \prime}\right)_{\mathfrak{S}_{s}}$ (ref. [4], Chap. I, $\$ 2$, No. 2). Therefore one must show $\left(\mathfrak{H}^{\prime \prime}\right)_{\mathfrak{S}_{s}} \supset \mathfrak{B}\left(\mathfrak{S}_{s}\right)$. According to Theorem 2 , $(\mathfrak{A})_{\mathfrak{F}_{s}}$ and thus $\left(\mathfrak{Q}^{\prime \prime}\right)_{\mathfrak{S}_{s}}$ contains the algebra $\mathfrak{C}\left(\mathfrak{G}_{s}\right)$ of all completely continuous operators on $\mathfrak{G}_{s}:\left(\mathfrak{U}^{\prime \prime}\right)_{\mathfrak{F}_{s}} \supset \mathfrak{C}\left(\mathfrak{S}_{s}\right)$. But because $\left(\mathfrak{Q}^{\prime \prime}\right)_{\mathfrak{S}_{s}}$ is a von Neumann algebra on the Hilbert space $\mathfrak{G}_{s}$, it follows

$$
\left(\mathfrak{U}^{\prime \prime}\right)_{\mathfrak{S}_{s}} \equiv\left(\mathfrak{U}^{\prime \prime}\right)_{\mathfrak{S}_{s}}^{\prime \prime}>\mathfrak{C}\left(\mathfrak{S}_{s}\right)^{\prime \prime}=\mathfrak{B}\left(\mathfrak{G}_{s}\right)
$$

This proof of Theorem 2 decisively uses the special structure of the von Neumann algebra $\Re$, together with Theorem 2 . An entirely different, but somewhat more complicated proof can be given using methods which will be developed later in connection with Theorem 5 (for details see $\S 3)$ : Let be $f_{i}(g), i=1,2 \ldots$ a $\delta$-sequence on $(\mathfrak{S}, X$ an arbitrary operator in $\mathfrak{R}$, and $X\left(f_{i}\right)=\int \mathbf{V}_{g} X f_{i}(g) d g$ with the left-invariant integral on $\mathfrak{G}$. 
Then one has $X\left(f_{i}\right) \in \mathfrak{A}$ and

$$
\lim _{i \rightarrow \infty} X\left(f_{i}\right)=X \quad \text { (ultrastrong convergence), }
$$

i.e., $\mathfrak{R}$ is the ultrastrong closure of $\mathfrak{A}$. The rest of Theorem 3 follows again from Dixmier [4] (Chap. I, § 3, No. 4).

Corollary. Any Hermitean operator in $\mathfrak{R}$ is a weak (ultraweak, strong, ultrastrong) accumulation point of Hermitean operators from $\mathfrak{A}$ (i.e., of observables).

Proof. Let $\mathfrak{R}$, resp. $\mathfrak{M}$, be the set of all Hermitean operators in $\mathfrak{A}$, resp. $\mathfrak{R}$, and denote by $\mathfrak{Z}_{\mathrm{w}}, \mathfrak{R}_{\text {uw }}, \mathfrak{T}_{\text {st }}, \mathfrak{T}_{\text {ust }}$ the closure of $\mathfrak{R}$ in the weak, ultraweak, strong, and ultrastrong topology, respectively. One has to show

$$
\mathfrak{R} \subset \mathfrak{R}_{\mathrm{w}}, \quad \mathfrak{R} \subset \mathfrak{R}_{\mathrm{uw}}, \quad \mathfrak{R} \subset \mathfrak{R}_{\mathrm{st}}, \quad \mathfrak{M} \subset \mathfrak{R}_{\mathrm{ust}} .
$$

Since the involution $X \rightarrow X^{*}$ is weakly continuous, $\mathscr{2}_{\mathrm{w}}$ consists of Hermitean operators, and since $\mathfrak{R}$ is weakly closed, one has

$$
\mathfrak{T}_{\mathrm{w}} \subset \mathfrak{R} \text {. }
$$

The known relations between the various operator topologies (Drxmren [4], Chap. I, § 3, No. 2) imply

$$
\mathfrak{T}_{\text {ust }} \subset \mathfrak{T}_{\mathrm{st}} \subset \mathfrak{T}_{\mathrm{w}}, \quad \mathfrak{R}_{\mathrm{ust}} \subset \mathfrak{T}_{\mathrm{uw}} \subset \mathfrak{R}_{\mathrm{w}} \text {. }
$$

On the other hand, according to Theorem 3 any $A \in \mathfrak{Z}$ is an ultraweak accumulation point of operators $X \in \mathfrak{A}$ and thus, by ultraweak continuity of the involution, also an ultraweak accumulation point of the Hermitean operators $\frac{1}{2}\left(X^{*}+X\right) \in \mathcal{R}$, i.e.,

$$
\mathfrak{T}_{\mathrm{uw}} \supset \mathfrak{R} \text {. }
$$

Eqs. (4), (5), and (6) together imply

$$
\mathfrak{Z}_{\mathrm{w}}=\mathfrak{Q}_{\mathrm{uw}}=\mathfrak{M} \text {. }
$$

The set $\mathfrak{Z}$ is convex, therefore its closures $\mathfrak{Z}_{\text {st }}$ and $\mathfrak{Z}_{\text {ust }}$ are convex too. According to Dixmier [5], $\mathfrak{T}_{\text {st }}$ resp. $\mathfrak{T}_{\text {ust }}$ are then also weakly resp. ultraweakly closed, thus

$$
\mathfrak{R}_{\text {st }} \supset \mathfrak{Q}_{\mathrm{w}}, \quad \mathfrak{R}_{\text {ust }} \supset \mathfrak{R}_{\text {uw }} \text {. }
$$

This implies, together with (5) and (7),

q.e.d.

$$
\mathfrak{R}_{\mathrm{st}}=\mathfrak{2}_{\mathrm{w}}=\mathfrak{R}_{\mathrm{ust}}=\mathfrak{R}_{\mathrm{uw}}=\mathfrak{M} \text {, }
$$

A shorter proof follows by using a $\delta$-sequence $f_{i}(g), i=1,2 \ldots$ (see $\S 3$ ). For any $A \in \mathfrak{M}$, the $A\left(f_{i}\right)=\int \mathbf{V}_{g} A f_{i}(g) d g$ are Hermitean operators belonging to $\mathfrak{A}$, and $\lim _{i \rightarrow \infty} A\left(f_{i}\right)=A$ (ultrastrongly) implies

$$
2 \mathfrak{r}_{\text {ust }} \supset \mathfrak{M} \text {. }
$$


From this and (5) follows also

$$
\mathfrak{R}_{\mathrm{w}} \supset \mathfrak{M}, \quad \mathfrak{R}_{\mathrm{uw}} \supset \mathfrak{M}, \quad \mathfrak{P}_{\mathrm{st}} \supset \mathfrak{M} \text {. }
$$

Conversely, Theorem 3 follows also from the Corollary. Any operator $X \in \mathfrak{R}$ can be written as $A+i B$ with Hermitean $A$ and $B ; A$ and $B$ however - and thus $X$ - can, according to the Corollary, be approximated weakly (ultraweakly, strongly, ultrastrongly) by operators from $\mathfrak{A}$.

\section{The Algebra of Quasilocal Observables}

The methods of $\S 2$ will be applied in this section to axiomatic quantum field theory. This amounts to a nontrivial generalization, since now the algebraic structure becomes more complicated: Instead of the single von Neumann algebra $\mathfrak{R}$ of $\S 2$, local von Neumann algebras $\mathfrak{R}_{C}$ are given for all finite open space-time regions $C$. Furthermore, in contrast to $\Re$ the $\Re_{C}$ are not invariant with respect to the representation $U_{g}$ in $\mathfrak{S}$ of the symmetry group $\mathfrak{G}$, the latter going to be identified with the proper inhomogeneous Lorentz (or space-time translation) group.

The following postulates are assumed (compare, e.g., Arakr [6]):

I. For any finite open space-time region $C$, a von Neumann algebra $\mathfrak{R}_{C}$ of operators on Hilbert space $\mathfrak{G}$ is given.

II. If $C=\bigcup_{i \in I} C_{i}$ with any index set $\mathrm{I}$, then

$$
\mathfrak{R}_{C}=\left\{\bigcup_{i \in I} \mathfrak{R}_{C_{i}}\right\}^{\prime \prime} \text {. }
$$

Corollary. $\mathfrak{R}_{C_{1}} \subset \mathfrak{R}_{C_{2}}$ if $C_{1} \subset C_{2}$ (isotony).

III. A continuous unitary representation up to a factor $U(a, A)$ of the inhomogeneous Lorentz group $\mathfrak{G}$ in $\mathfrak{S}$ is given with

$$
U(a, \Lambda) \mathfrak{R}_{C} U^{-1}(a, \Lambda)=\mathfrak{R}_{C_{(a, \Lambda)}} .
$$

(Here $C_{(a, A)}$ denotes the region generated from $C$ by the transformation $x \rightarrow \Lambda x+a$.) -

The transformation $\mathbf{V}(a, \Lambda)$, for any operator $X$ on $\mathfrak{S}$, is defined as above by

$$
\mathbf{V}(a, \Lambda) X=U(a, \Lambda) X U^{-1}(a, \Lambda) .
$$

The $\mathbf{V}(a, \mathcal{A})$ form again a representation of the group $\mathfrak{G}$ on $\mathfrak{Z}(\mathfrak{H})$. Because of Postulate III, however, they are not automorphisms of the algebras $\mathfrak{R}_{C}$. The set of operators $X \in \mathfrak{R}_{C}$ with

$$
\lim _{(a, \Lambda) \rightarrow(0,1)}\|\mathbf{V}(a, \Lambda) X-X\|=0
$$

will be denoted by $\mathfrak{A}_{C}$. In complete analogy to Theorem 1 one proves:

Theorem $4 . \mathfrak{A}_{C}$ is a concrete $C^{*}$-algebra.

In the following, $\mathfrak{A}_{C}$ is called the algebra of local observables for the region $C$. The Hermitean operators in $\mathfrak{A}_{C}$ shall represent all possible measurements in the space-time region $C$. 
Isotony of the mapping $C \rightarrow \mathfrak{R}_{C}$ evidently implies isotony of $C \rightarrow \mathfrak{A}_{C}$. Postulate III leads to

$$
\mathbf{V}(a, \Lambda) \mathfrak{A}_{C}=\mathfrak{A}_{C_{(a, \Lambda)}} .
$$

Finally, the $\mathfrak{A}_{C}$ satisfy the usual postulate of local commutativity if one assumes the corresponding property of the $\mathfrak{R}_{C}$.

With the same methods as in $\S 2$ one could formally prove an analogue to Theorem 2 for the local algebra of observables $\mathfrak{A}_{C}$. This, however, makes no sense: No algebra $\mathfrak{R}_{C}$ should contain any completely continuous operator except zero (MisRa, Borcirers [7]).

But again one can prove:

Theorem 5. The local algebra of observables $\mathfrak{A}_{C}$ is weakly (ultraweakly, strongly, ultrastrongly) dense in the local von Neumann algebra $\mathfrak{R}_{C}$, i.e., $\mathfrak{A}_{C}^{\prime \prime}=\mathfrak{R}_{C}$.

As in $\S 2$, this implies:

Corollary. Any Hermitean operator in $\mathfrak{R}_{C}$ is a weak (ultraweak, strong, ultrastrong) accumulation point of local observables belonging to the region $C$, i.e., of Hermitean operators from $\mathfrak{A}_{C}$.

Theorem 5 is more complicated to prove than the analogous Theorem 3 . The algebraic structure of $\mathfrak{R}_{C}$, in contrast to $\mathfrak{R}$, is not explicitely known, and especially an useful analogue of Theorem 2 is missing here. The method of proof here applied, using $\delta$-sequences on Lie groups, has been developed by ARAKr [6] for the special case of the translation group.

Definition. Let be $\mathfrak{G}$ any Lie group and $\int \ldots d g$ the left-invariant integral on $\mathfrak{G}$. A sequence $f_{i}(g), i=1,2 \ldots$ of continuous nonnegative functions with compact carriers $\operatorname{Supp} f_{i}$ on $\mathfrak{G}$ is called a $\delta$-sequence, if

and

$$
\int f_{i}(g) d g=1 \text { for all } i=1,2 \ldots
$$

$$
\operatorname{Supp} f_{i} \rightarrow e \text { for } i \rightarrow \infty \text {. }
$$

(The last statement means, more precisely: For any neighborhood $U(e)$ of the unit element $e$ on $\mathfrak{G}$ there exists a number $N$ such that Supp $f_{i} \subset U(e)$ for all $i>N$.) -

For any continuous function $f(g)$ and any $\delta$-sequence $f_{i}(g)$ on $\mathfrak{S}$ then

$$
\lim _{i \rightarrow \infty} \int f(g) f_{i}(g) d g=f(e),
$$

thus justifying the name " $\delta$-sequence". The existence of such $\delta$-sequences and property (11) are well known (see, e.g., Dixmier [8]).

The subsequent lemma will be decisive for the following:

Lemma. Let $U_{g}$ be a continuous unitary representation up to a factor of the Lie group $\mathfrak{S}$ in Hilbert space $\mathfrak{S}$. Furthermore, let $f_{i}(g), i=1,2 \ldots$ be a $\delta$-sequence on $\mathfrak{G}$ and $X$ an operator from a von Neumann algebra $\mathfrak{T}$ on $\mathfrak{S}$ with $\mathbf{V}_{g} X=U_{g} X U_{g}^{-1} \in \mathfrak{T}$ for all $g \in \operatorname{Supp} f_{i}, i=1,2 \ldots$ Then the following statements hold: 
1. The $X\left(f_{i}\right)=\int \mathbf{V}_{g} X f_{i}(g) d g, i=1,2 \ldots$ are bounded operators on $\mathfrak{S}$ with $\left\|X\left(f_{i}\right)\right\| \leqq\|X\|$. For Hermitean $X$, the $X\left(f_{i}\right)$ are Hermitean too.

2. $X\left(f_{i}\right) \in \mathfrak{T}, i=1,2 \ldots$.

3. $\lim _{g \rightarrow e}\left\|\mathbf{V}_{g} X\left(f_{i}\right)-X\left(f_{i}\right)\right\|=0, i=1,2 \ldots$.

4. $\lim _{i \rightarrow \infty} X\left(f_{i}\right)=X$ (ultrastrong convergence).

Proof. 1. The equation

$$
\langle\varphi, \psi\rangle=\int\left(\varphi, \mathbf{V}_{g} X \psi\right) f_{i}(g) d g \text { for all } \varphi, \psi \in \mathfrak{G}
$$

defines a bilinear form on $\mathfrak{G}$, which is bounded by

$$
|\langle\varphi, \psi\rangle| \leqq \int\left|\left(\varphi, \mathbf{V}_{g} X \psi\right)\right| f_{i}(g) d g \leqq\|\varphi\| \cdot\|X\| \cdot\|\psi\| \cdot
$$

For Hermitean $X,\langle\varphi, \psi\rangle=\langle\psi, \varphi\rangle^{*}$. Therefore

defines an operator

$$
\langle\varphi, \psi\rangle=\left(\varphi, X\left(f_{i}\right) \psi\right)
$$

$$
X\left(f_{i}\right)=\int \mathbf{V}_{g} X f_{i}(g) d g
$$

with $\left\|X\left(f_{i}\right)\right\| \leqq\|X\|$, which is Hermitean if $X$ is.

2. By definition of $X\left(f_{i}\right)$,

$$
\left(\varphi, Y X\left(f_{i}\right) \psi\right)=\int\left(\varphi, Y \mathbf{V}_{g} X \psi\right) f_{i}(g) d g
$$

for any $Y \in \mathfrak{T}^{\prime}$, and since $\mathbf{V}_{g} X \in \mathfrak{T}$ for all $g \in \operatorname{Supp} f_{i}$, one can transform the right hand side into

$$
\int\left(\varphi,\left[\mathbf{V}_{g} X\right] Y \psi\right) f_{i}(g) d g=\left(\varphi, X\left(f_{i}\right) Y \psi\right) .
$$

Therefore $X\left(f_{i}\right) Y=Y X\left(f_{i}\right)$ for all $Y \in \mathfrak{T}^{\prime}$, i.e., $X\left(f_{i}\right) \in \mathfrak{T}^{\prime \prime}=\mathfrak{T}$.

3. One has

$$
\begin{aligned}
\mathbf{V}_{g} X\left(f_{i}\right) & =\int \mathbf{V}_{g} \mathbf{V}_{h} X f_{i}(h) d h=\int \mathbf{V}_{g h} X f_{i}(h) d h \\
& =\int F(h, g) d h,
\end{aligned}
$$

and according to the left-invariance of the integral the last term equals

So one obtains

$$
\int F\left(g^{-1} h, g\right) d h \equiv \int \mathbf{V}_{h} X f_{i}\left(g^{-1} h\right) d h .
$$

$$
\begin{aligned}
\left\|\mathbf{V}_{g} X\left(f_{i}\right)-X\left(f_{i}\right)\right\| & =\left\|\int \mathbf{V}_{h} X\left[f_{i}\left(g^{-1} h\right)-f_{i}(h)\right] d h\right\| \leqq \\
& \leqq\|X\| \int\left|f_{i}\left(g^{-1} h\right)-f_{i}(h)\right| d h .
\end{aligned}
$$

Since $f_{i}(g)$ is a continuous function with compact carrier, it is also uniformly continuous. Therefore, if $g \rightarrow e$, the integrand $\mid f_{i}\left(g^{-1} h\right)-$ $-f_{i}(h) \mid$ in the last term tends to zero uniformly in $h$, whereas the integration volume (i.e., the region with $f_{i}\left(g^{-1} h\right)-f_{i}(h) \neq 0$ ) contracts to Supp $f_{i}$, with $\int_{\text {Supp } f_{i}} d h<\infty$ because Supp $f_{i}$ is compact. This implies statement 3 . of the Lemma. 
4. By definition of $X\left(f_{i}\right)$, for any $\varphi \in \mathfrak{S}$ one has

$$
\begin{aligned}
\left\|\left[X\left(f_{i}\right)-X\right] \varphi\right\| & =\operatorname{Sup}_{\|\psi\|=1}\left|\left(\psi,\left[X\left(f_{i}\right)-X\right] \varphi\right)\right| \\
& =\operatorname{Sup}_{\|\psi\|=1}\left|\int\left(\psi,\left[\mathbf{V}_{g} X-X\right] \varphi\right) f_{i}(g) d g\right| \leqq \\
& \leqq \operatorname{Sup}_{\|\psi\|=1}\|\psi\| \cdot \int\left\|\left[\mathbf{V}_{g} X-X\right] \varphi\right\| f_{i}(g) d g \leqq \\
& \leqq \operatorname{Sup}_{g \in \operatorname{Supp} f_{i}}\left\|\left[\mathbf{V}_{g} X-X\right] \varphi\right\| .
\end{aligned}
$$

$\mathbf{V}_{g} X$ being a strongly continuous function of $g(\S 2$, eq. (1)), the last term vanishes for $i \rightarrow \infty$ since $\operatorname{Supp} f_{i} \rightarrow e$. Thus

$$
\lim _{i \rightarrow \infty}\left\|\left[X\left(f_{i}\right)-X\right] \varphi\right\|=0 \text { for all } \varphi \in \mathfrak{H},
$$

i.e., the $X\left(f_{i}\right)$ converge strongly to $X$. Moreover, according to 1 . one has $\left\|X\left(f_{i}\right)\right\| \leqq\|X\|$ for all $i$, i.e., all operators $X\left(f_{i}\right)$ belong to a common sphere $\|Y\| \leqq$ const. in $\mathfrak{B}(\mathfrak{S})$. Within such spheres, however, the ultrastrong operator topology coincides with the strong one (DixmIER [4], Chap. I, $\S 3$, No. 2), i.e., the $X\left(f_{i}\right)$ converge to $X$ even ultrastrongly.-

With the help of this Lemma, applied to $\mathfrak{I}=\mathfrak{R}$, the alternative proof of Theorem 3 sketched in $\S 2$ follows immediately.

Proof of Theorem 5. One has to prove $\mathfrak{R}_{C}=\mathfrak{A}_{C}^{\prime \prime}$, and it suffices to show $\mathfrak{R}_{C} \subset \mathfrak{U}_{C}^{\prime \prime}$ since $\mathfrak{U}_{C}^{\prime \prime} \subset \mathfrak{R}_{C}$ immediately follows from $\mathfrak{A}_{C} \subset \mathfrak{R}_{C}$.

Consider an arbitrary open region $B$ with $\bar{B} \subset C .(\bar{B}$ denotes the closure of $B$.) The set of all transformations $(a, \Lambda)$ with $\overline{\boldsymbol{B}}_{(a, \Lambda)} \subset C$ is then a neighborhood $C-B$ of the unit element $e=(0,1)$ of the inhomogeneous Lorentz group $\mathfrak{H}$. Then choose a $\delta$-sequence $f_{i}(a, \Lambda)$ on $\mathfrak{G}$ such that $\operatorname{Supp}_{i} \subset C-B$ for all $i=1,2 \ldots$.

Let $X$ be any operator from $\mathfrak{R}_{B}$. The Postulates II and III imply $\mathbf{V}(a, \Lambda) X \in \mathfrak{R}_{C}$ for all $(a, \Lambda) \in C-B$, thus also for all $(a, \Lambda) \in \operatorname{Supp} f_{i}$, $i=1,2 \ldots$. Then the above Lemma can be applied with $\mathfrak{I}=\mathfrak{R}_{C}$. From the statements 2. and 3. of the Lemma,

$$
X\left(f_{i}\right)=\int \mathbf{V}(a, \Lambda) X f_{i}(a, \Lambda) d(a, \Lambda) \in \mathfrak{A}_{C},
$$

and from 4., $\lim _{i \rightarrow \infty} X\left(f_{i}\right)=X$ (ultrastrongly). Therefore $X$, and thus the whole algebra $\mathfrak{R}_{B}$, belongs to the ultrastrong closure of $\mathfrak{A}_{C}$, i.e., to $\mathfrak{P}_{C}^{\prime \prime}$.

On the other hand, the region $C$ can be represented as the union of all regions $B$ with $\bar{B} \subset C$, and Postulate II implies

$$
\mathfrak{R}_{C}=\left\{\bigcup_{\bar{B}} \subset \mathfrak{R}_{B}\right\}^{\prime \prime} .
$$

Together with the relation $\mathfrak{R}_{B} \subset \mathfrak{A}_{C}^{\prime \prime}$ just proved this implies $\mathfrak{R}_{C} \subset \mathfrak{A}_{C}^{\prime \prime}$.-

Now, all local algebras of observables $\mathfrak{A}_{C}$ for finite open regions $C$ can be combined to a single $C^{*}$-algebra $\mathfrak{A}$, the so called algebra of quasilocal observables (or shortly, quasilocal algebra). Namely, let $\mathfrak{A}$ be the closure 
of the set of operators $\mathfrak{B}=\bigcup_{C \text { finite }} \mathfrak{A}_{C}$ with respect to the uniform operator topology. The set $\mathfrak{Z}$ is a symmetric algebra of bounded operators: Choose $X$ and $Y$ arbitrarily from $\mathfrak{B}$, i.e., $X \in \mathfrak{A}_{C_{1}}$ and $Y \in \mathfrak{A}_{C_{2}}$ with suitable finite regions $C_{1}$ and $C_{2}$. As implied by isotony, the local algebra $\mathfrak{A}_{C_{1} \cup C_{2}}$ contains both $\mathfrak{A}_{C_{1}}$ and $\mathfrak{A}_{C_{2}}$. Thus $\lambda X, X^{*}, X+Y$, and $X Y$ belong to $\mathfrak{A}_{C_{1} \cup C_{2}}$ and to $\mathfrak{B} \supset \mathfrak{A}_{C_{1} \cup C_{2}}$. The uniform closure $\mathfrak{A}$ of $\mathfrak{Z}$ is then indeed a concrete $C^{*}$-algebra.

By eq. (10), the transformations $\mathbf{V}(a, \Lambda)$ are a representation of $(\mathfrak{H}$ by automorphisms of the algebra $\mathfrak{B}$, and since $\|\mathbf{V}(a, \Lambda) X\|=\|X\|$, the set of uniform accumulation points of $\mathfrak{Z}$ is also transformed into itself, i.e., the $\mathbf{V}(a, \Lambda)$ are automorphisms of the quasilocal algebra $\mathfrak{A}$ too. The continuity of $\mathrm{V}(a, \Lambda)$ expressed by $(9)$ is similarly transferred from $\mathfrak{Z}$ to its uniform closure $\mathfrak{A}$.

If the $\mathfrak{R}_{C}$, and thus also the $\mathfrak{A}_{C}$, do not contain completely continuous operators [7], then the same must hold for the quasilocal algebra 2. This follows easily with the help of a theorem from Rresz-NaGY [9].

An entirely analogous construction of $C^{*}$-algebras $\widetilde{\mathfrak{A}}_{C}$ and $\widetilde{\mathfrak{A}}$ can be performed by using, instead of the inhomogeneous Lorentz group, the space-time translation group only. Since the latter is a subgroup of the former, one has $\widetilde{\mathfrak{U}}_{C} \supset \mathfrak{I}_{C}$ and $\widetilde{\mathfrak{U}} \supset \mathfrak{A}$. The algebras $\widetilde{\mathfrak{I}}_{C}$ and $\widetilde{\mathfrak{U}}$, however, can also be constructed if one assumes, instead of Postulate III, only translation invariance.

Replacing the concrete $C^{*}$-algebra $\mathfrak{A}$ and its local subalgebras $\mathfrak{A}_{C}$ constructed here by the corresponding abstract $C *$-algebras, one arrives at a purely algebraic structure. Such an abstract formulation of local field theory has been proposed by HAAG and KASTLER [1]. The quasilocal algebra $\mathfrak{A}$ given here fulfills all postulates of ref. [1] if, besides Postulates I to III, also local commutativity of the algebras $\mathfrak{R}_{C}$ is assumed. Thus the above construction of $\mathfrak{A}_{C}$ and $\mathfrak{A}$ can also be interpreted as a prescription how to associate abstract $C^{*}$-algebras with a given concrete (i.e., Hilbert space) field theory.

\section{Remarks on Algebraic Spectrum Conditions}

Besides satisfying the postulates of HAAG and KASTLER [1], the quasilocal algebra $\mathfrak{A}$ constructed above has, by its very definition, a further important property expressed by eq. (9). The representation of the inhomogeneous Lorentz group $\mathfrak{5}$ by isometric operators $\mathbf{V}(a, \Lambda)$ on the Banach space $\mathfrak{A}$ is, according to (9), strongly continuous. In this sense, eq. (9) is an "algebraic" analogue of the strong continuity of the representation of $\mathfrak{G}$ by unitary operators $U(a, \Lambda)$ on Hilbert space $\mathfrak{H}$ 8 Commun. math. Phys., Vol. 7 
(Postulate III). The usual Hilbert space spectrum condition of field theory is, by Stone's theorem, connected with the strong continuity of $U(a, \Lambda)$. "Algebraic" spectrum conditions, i.e., corresponding assumptions for the automorphisms $\mathbf{V}(a, \Lambda)$ of $\mathfrak{A}$, can now be formulated with the help of eq. (9).

Since only the translations $\mathbf{V}(a, 1)=\mathbf{V}(a)$ are needed in this connection, one could use instead of (9) the weaker requirement

$$
\lim _{a \rightarrow 0}\|\mathbf{V}(a) X-X\|=0 \text { for all } X \in \mathfrak{Q} \text {, }
$$

or even treat instead of $\mathfrak{A}$ the larger algebra $\widetilde{\mathfrak{A}}$ mentioned above.

Montvay [2] translates, almost literally, the usual spectrum condition into an algebraic one by replacing the infinitesimal generators $P_{i}$ of $U(a, 1)=U(a)$ on $\mathfrak{G}$ by the corresponding generators $\mathbf{P}_{i}$ of $\mathbf{V}(a)$ on $\mathfrak{A}$. The condition thus obtained implies the Hilbert space spectrum condition for all irreducible translation-invariant representations of $\mathfrak{U}[2]$.

Another algebraic spectrum condition has been formulated by Doplicher [3]. From it follows the existence of at least one translationinvariant representation with the Hilbert space spectrum condition; furthermore, this representation can also be chosen to be irreducible [3]. Montray's condition is thus the stronger one, and is perhaps even too strong [10].

We will not go into more details here, but only add a somewhat trivial remark. In ref. [3], no continuity assumption like (9) or (12) seems to be needed at all. Without such an assumption, however, two difficulties appear. First, expressions like $\int \mathbf{V}(x) A z(x) d^{4} x$ with $A \in \mathfrak{A}$ and a summable function $z(x)$ which are used in ref. [3] are then not defined as elements of $\mathfrak{A}$. (The isometry of $\mathbf{V}(x)$ is clearly not sufficient for this.) Secondly, no continuity properties of the unitary representation $U(a)$ in the Hilbert space $\mathfrak{S}$ of a translation-invariant representation of $\mathfrak{A}$, and thus no spectral decomposition $U(a)=\int e^{i p a} d E(p)$, can be deduced. Both difficulties disappear if eq. (12) is assumed to hold.

A continuity assumption like (12) seems thus to be necessary also for DoPLICHER's spectrum condition. This is intuitively expected to be true for any attempt to formulate spectrum conditions algebraically.

Acknowledgments. The author would like to express his gratitude to Professor G. LUDWIG for suggesting this investigation, and to Professor D. KASTLER for numerous helpful comments. He is also indebted to Mr. P. RosentHaL for reading the manuscript, and to the Deutsche Forschungsgemeinschaft for financial support. 


\section{References}

1. HaAG, R., and D. Kastler: J. Math. Phys. 5, 848 (1964).

2. Montvay, I.: Nuovo Cimento 40 A, 121 (1965).

3. Doplicher, S.: Commun. Math. Phys. 1, I (1965).

4. Dixmier, J.: Les algèbres d'opérateurs dans l'espace Hilbertien (Algèbres de von Neumann). Paris: Gauthier-Villars 1957.

5. - Ann. Math. 51, 387 (1950), Théorème 5.

6. Araki, H.: Einführung in die Axiomatische Quantenfeldtheorie (unpublished lecture notes); Zürich 1961/62.

7. Mrsra, B.: Helv. Phys. Acta 38, 189 (1965). - Borchers, H. J.: Commun. Math. Phys. 4, 315 (1967).

8. Dixmier, J.: Les $C^{*}$-algèbres et leurs représentations; $§ 13$, No. 13.2.5. Paris: Gauthier-Villars 1964.

9. Riesz, F., and B. Sz.-NAGY: Functional Analysis; Chap. IX, No. 135, second Theorem. New York: Ungar 1955.

10. Kastler, D.: Private communication. 